\title{
A STUDY ON THE DEVELOPMENT OF PATH FINDING ALGORITHM FOR PASSENGER FLOW IN RAILWAY STATION
}

\author{
SEONGWON NAM \\ Korea Railroad Research Institute, Korea
}

\begin{abstract}
A computational path finding method has been developed to simulate the flow of passengers at railway transit stations. The concept of computational method is very similar to particle-laden flow. The basic procedures are as follows. Like general computational fluid dynamics, the computational domain is divided into meshes and potential values are calculated for each cell by providing boundary conditions for inlet and outlet. The path line is then calculated according to the potential value. The path line obtained is the basic moving path, but it is an algorithm that finds a new path by changing the path according to the situation. Representative situations in which passengers may change routes at railway stations are as follows. That is, there is a slow pedestrian in the direction that the pedestrian is going to move or encounter a pedestrian in the opposite direction. According to a specific rule-based system, we developed an algorithm to find the path to change, and the main factors such as walking speed, viewing angle, straightness, walking on the right, etc. were considered. The analysis results show that pedestrians do not move along the shortest paths but change their paths from time to time. Through the analysis of passenger flow, it is expected that it can be applied to the optimal structural design of railway stations and the convenient flow of passenger.
\end{abstract}

Keywords: computational method, crowd flow, path finding, railway station, rule-based system.

\section{INTRODUCTION}

Metropolitan railway stations, terminals, multi-purpose facilities and stadiums are representative crowded places. The analysis of crowd flow is important not only for the improvement of user's path but also for the prevention of safety accidents. There are a number of factors that affect how people choose to travel route from the origin to the destination. If the path is known in advance, people will use the shortest path, but if it is a strange one, visibility for structure and various guide signs will work. In this study, walking speed, the angle of view, straightness and walking on the right were considered as major factors for path finding. The walking speed varies according to age or health condition or disability, and the straightness will change depending on the psychological state, for example, whether it is free, normal or urgent. The range of path selection depends on the angle of view. In other words, the range of path search will vary depending on whether pedestrians have a wide view or a narrow view. In computational analysis, the key is how to express these qualitative parameters discretely and quantitatively. The aforementioned parameters can be given a specific value to express various situations quantitatively. If a disaster such as a fire occurs, the degree of chaos will be severe, so there is almost no right-hand traffic, and the straightness and the viewing angle can be expressed as being smaller. By giving the straight probability value and the angle of view small, it was expressed that the chaos parameter, which is a qualitative element, is large.

Many methods have been proposed to study the crowd flow dynamics. Nam proposed new computational method for passenger flow by using potential path line concept [1,2], Helbing used the social force model to study the movement of people in the panic state [3-4]. There are also examples using cellular automata model [6-8], lattice gas model [9-12], agentbased model [13] and forces model [14]. Each of these analysis methods has advantages and 
disadvantages and is appropriately applied according to the calculation model. Details of the potential path line method are described in references [1,2]. The analysis results show that pedestrians do not necessarily choose the shortest path and often change the path. In this study, simulations were performed on the ticket gate models of railway stations, and the path finding performance was simulated for the representative maze model. As a result of the comparison between ticket gate models of railway station, the walking time changed greatly depending on how much the path line overlaps. The path finding of passengers using the potential path line method is also applicable to the complex maze models.

\section{SIMULATION ON TICKET GATE MODELS OF RAILWAY STATION}

The ticket gate models of railway station were set as follows. The size of the model is $15 \mathrm{~m}$ in width and length, and the number of ticket gate is 8 . The population flow rate at each inlet is six people per second. It flows for 7 seconds, and about 80 passengers enter each direction. A total of 160 people will cross. The average walking speed was assumed to be 1 to $1.4 \mathrm{~m} / \mathrm{s}$. The walking speed was randomly assigned to the people entering the entrance, and a person with a small value is a person with low mobility, and a large value can be treated as a person with a fast walking speed. Passengers marked with blue triangles enter from the lower two entrances, pass through the ticket gate and exit the upper left exit.

Passengers marked with red circle enter from the upper two entrances, pass through the ticket gates and exit the lower right exit. Model (a) allows eight gates to pass in both directions. That is, first come, first served. Model (b) is a structure in which four gates are installed in staggered directions, respectively. In model (c), four gates are arranged on the right side in each direction, and in model (d), the gates are arranged on the left side. In the models (b), (c) and (d), the gate direction is already determined, but in the model (a), the gate direction is determined by those who preoccupy the gate. In this study, we simulated how passenger flow changes with each gate type.

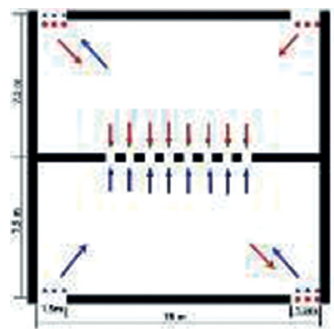

Gate model (a)

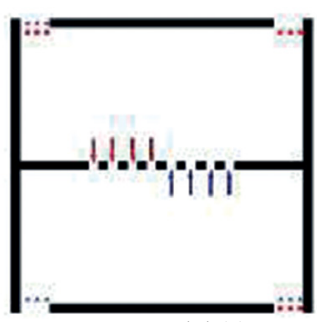

Gate model (c)

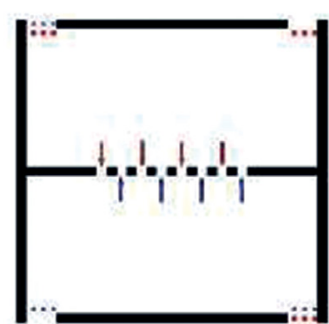

Gate model (b)

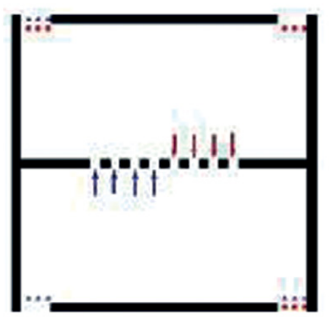

Gate model (d)

Figure 1: Ticket gate models. 


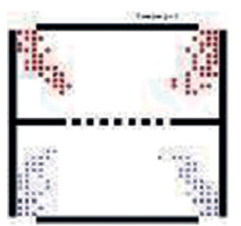

(a) $5 \mathrm{sec}$

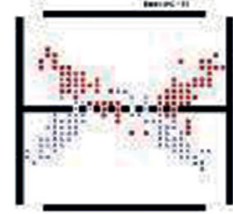

(b) $10 \mathrm{sec}$

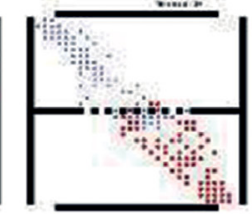

(c) $20 \mathrm{sec}$

Gate model (a)

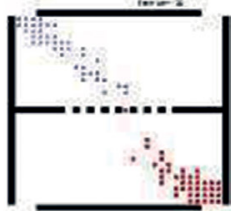

(d) $25 \mathrm{sec}$

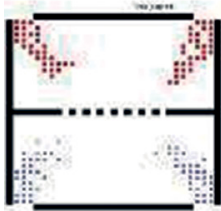

(a) $5 \mathrm{sec}$

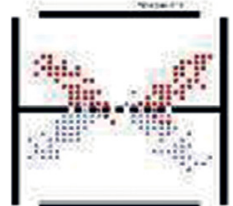

(b) $10 \mathrm{sec}$

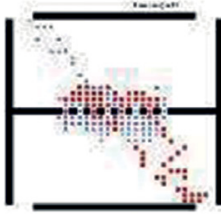

(c) $20 \mathrm{sec}$

(b)

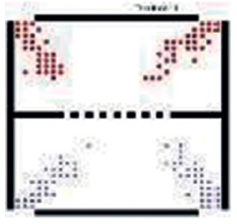

(a) $5 \mathrm{sec}$

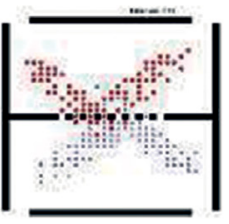

(b) $10 \mathrm{sec}$

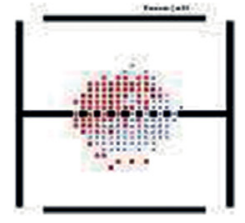

(c) $20 \mathrm{sec}$ Gate model (c)

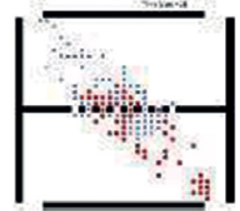

(d) $25 \mathrm{sec}$

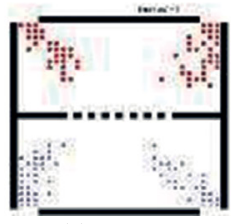

(a) $5 \mathrm{sec}$

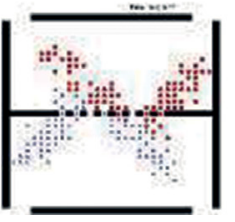

(b) $10 \mathrm{sec}$

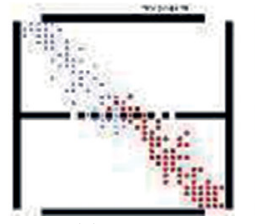

(c) $20 \mathrm{sec}$

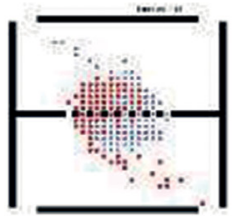

(d) $25 \mathrm{sec}$

Gate model (d)

Figure 2: Pedestrian distribution over time for gate model.

Figure 2 shows how pedestrian distribution over time for gate models. After 5 seconds, the distribution of people in the 4 models is not significantly different. After 10 seconds, the position of the pedestrians differs for each model. That is, pedestrians are crowded toward the gate direction of each model. After 20 seconds, pedestrians moved toward each exit for models (a) and (d), but stagnated near the gate for models (b) and (c). Although the number of gates in each direction is the same as four, it is considered that a difference occurs because the path lines of pedestrians are longer or cross each other. In model (a), for blue pedestrians, pedestrians entering from the lower left entrance form the path passing through the gates in left side to the upper left exit, and pedestrians entering from the lower right entrance go to the exit through the gates in the right middle. On the other hand, for red pedestrians, pedestrians entering from the upper right entrance form the path passing through the gates in right side to the lower right exit, and pedestrians entering from the upper left entrance go to the exit through the gates in the left middle. In the figures after 10 and 20 seconds, it is interesting that the pedestrian group using each gate changes over time. The user types per gate at 10 and 20 seconds are as follows. The user group type at 10 seconds, $\boldsymbol{\Delta} \boldsymbol{\Delta} \bullet \bullet \square \boldsymbol{\Delta} \bullet \bullet$ and at 20 
seconds, $\square \square \boldsymbol{\Delta} \bullet \boldsymbol{\Delta} \bullet \square \mathbf{\Delta}$. In model (b), blue pedestrians are set to use odd-numbered gates from the left, and red pedestrians are set to use even-numbered gates, so there is no change in pedestrian groups using gates throughout the simulation time. In model (c), according to the 'keep right' walking rule in Korea, it is set to use the gates in the right direction for each pedestrian group. Thus, red pedestrians use gates 1-4 from the left and blue pedestrians use gates 5-8. Because the paths overlap, the two groups of pedestrians experience heavy congestion near the gate.

Although 'keep right' is the principle, pedestrians entering the lower left entrance have longer paths to the exit and, in addition, overlap with the paths of pedestrians entering the upper right entrance. In model (d), in contrast to the 'keep right' rule, blue pedestrians use gates 1-4 on the left and red pedestrians use gates 5-8. While the opposite of the 'keep right' rule, for both groups, the paths are much smoother because the paths are shorter and do not overlap each other. Therefore, rather than unconditionally complying with the 'keep right', it is much more effective for the smooth flow to install the gate by analysing the usage patterns of pedestrians at each railway station. The congestion of the pedestrian flow for the 4 models can be seen by comparing the figures after 20 seconds. For each model, a comparison of six representative routes for each pedestrian group is shown in Fig. 3. It can be seen that the path of model (c) is much longer than other models and intersects with each other.

For each model, the travel time taken to walk from the inlet to the outlet is compared in Fig. 4. For each model, the results of 20 simulations are shown. In the quantitative analysis, for model (a), the minimum time required for all pedestrians to pass from the entrance to the exit is 33 seconds and the maximum time is 40.5 seconds, with an average of 35.7 seconds and a standard deviation of 2.21. For model (b), the minimum time is 40.5 seconds and the maximum time is 57.5 seconds, with an average of 47.55 seconds and a standard deviation of 5.83 . For model (c), the minimum time is 48 seconds and the maximum time is 58 seconds, with an average of 53.03 seconds and a standard deviation of 2.92. For model (d), the minimum time

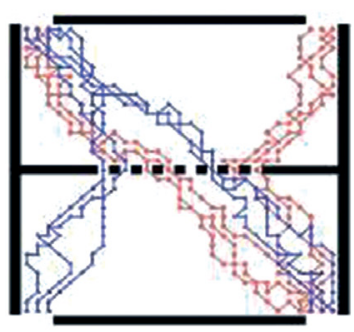

Gate model (a)

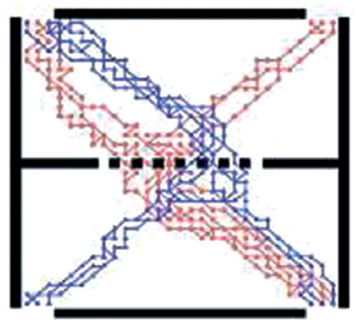

Gate model (c)

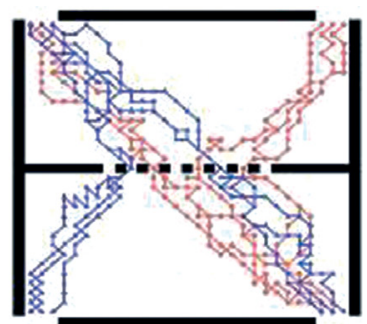

Gate model (b)

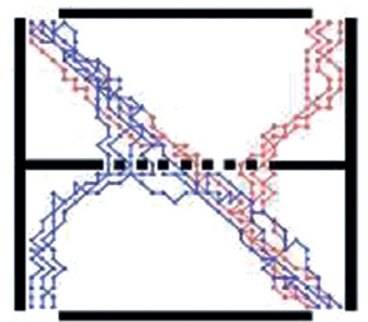

Gate model (d)

Figure 3: Comparison of representative paths. 


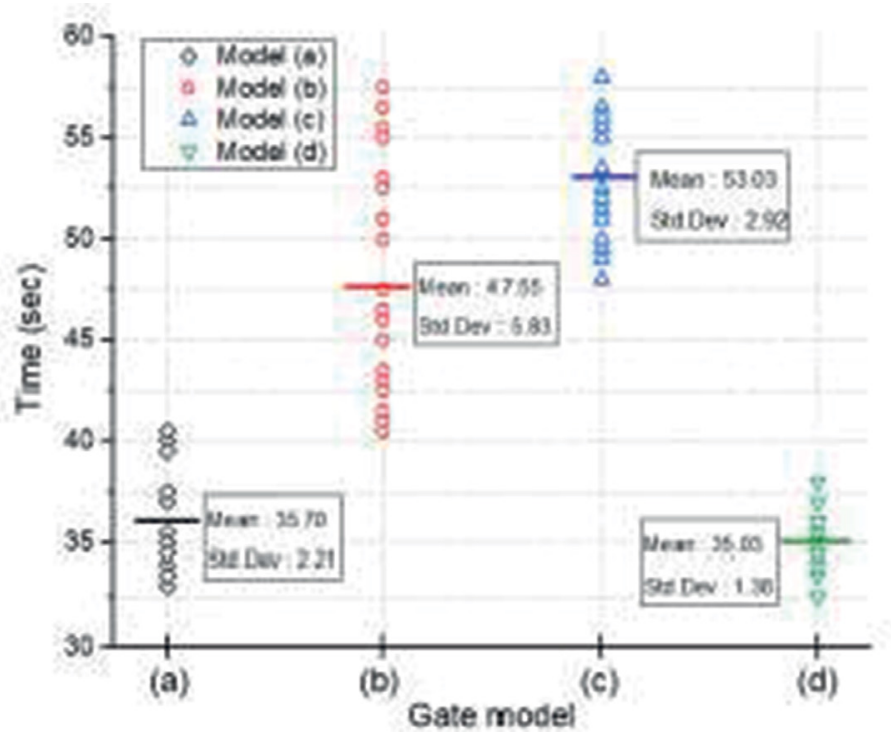

Figure 4: Travel time from inlet to exit.

is 32.5 seconds and the maximum time is 38 seconds, with an average of 35.03 seconds and a standard deviation of 1.36. Depending on how the gates are installed, the travel time varies significantly from case to case. As shown in the model (d), it can be seen that it is effective to install the gates so that the paths of pedestrians are short and do not overlap between the paths. If the paths of pedestrians are not known in advance, model (a) may be the second best. It is interesting to note that the model (c) with gates installed according to the 'keep right' rule has the longest travel time. From these results, it is important to predict the paths of pedestrians when designing a facility or building with crowds. The maximum and minimum travel time differences of the models (a), (b), (c) and (d) were 7.5, 17, 10 and 5.5 seconds, respectively. It can be seen that the gate path is not determined, or the more overlap between the paths, the greater the deviation.

\section{SIMULATION ON MAZE MODELS}

Figure 5 shows two maze models. The underground malls of the metropolitan railway stations are complex and crowded with people, making it look like a maze. Figure 5(a) is $15 \mathrm{~m}$ in width and length, and a maze is set randomly. Red pedestrians enter from the upper entrance and exit to the lower exit, and blue pedestrians enter from the lower entrance and exit to the upper exit. Figure 5(b) has a width of $15 \mathrm{~m}$ and a maze is set constantly. Red pedestrians enter from the upper entrance to the central exit, and blue pedestrians enter from the central entrance to the upper exit. In model (a), pedestrians have to decide which way to go on the crossroad, but in model (b), they will only find the path in the same direction. For the maze model, we analysed how the chaos parameter affects travel time.

The chaos parameter is a value between 0 and 1 , so chaos parameter 0 means no confusion at all, and chaos parameter 1 means very confusion. 'Confused' is a qualitative meaning that pedestrians are zigzag or right and left without going straight on the path. 


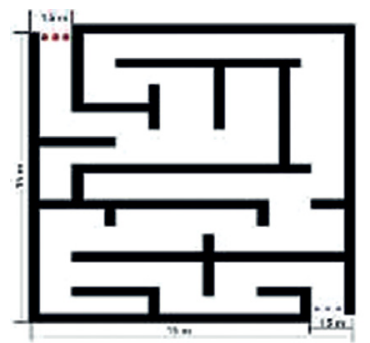

(a) Maze model (a)

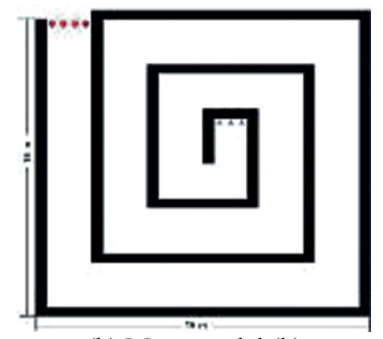

(b) Maze model (b)

Figure 5: Maze models.

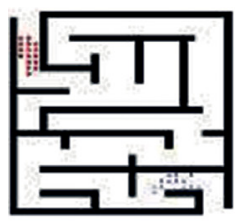

(a) $5 \mathrm{sec}$

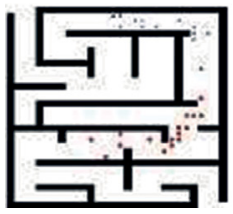

(e) $40 \mathrm{sec}$

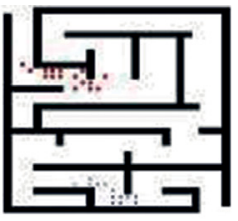

(b) $10 \mathrm{sec}$

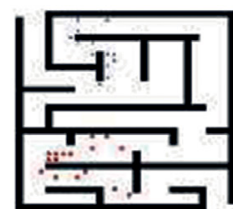

(f) $50 \mathrm{sec}$

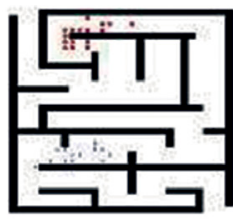

(c) $20 \mathrm{sec}$

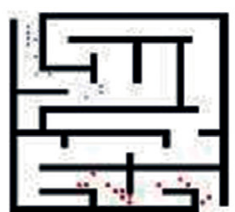

(g) $60 \mathrm{sec}$

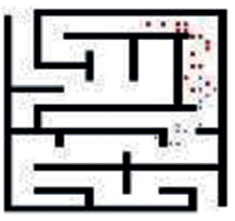

(d) $30 \mathrm{sec}$

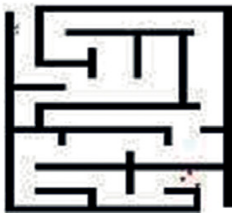

(h) $70 \mathrm{sec}$

Figure 6: Snapshots over time.

Model (a) is a structure that is influenced by chaos parameter a lot because there are many cases of chaos whether to go left or right on the route. On the other hand, model (b) has a structure that is less affected by chaos parameters because it only needs to turn in one direction. In the computational analysis, when the chaos parameter is $0.8,80 \%$ of people are supposed to move to the left and right cells without going directly in the cell of main moving direction. The fact that many people do not go right and left at the fork means that travel time is shortened. Even in such a small model, as the chaos parameter increases, the travel time increases by $12.5 \%$. In the event of a disaster, where the life and death split by a few seconds, the increase in evacuation time is very important. Figure 6 shows snapshots over time in model (a). Figure 7 shows percentage of normalized travel time for the chaos parameter. The time when the chaos parameter is 0 is set to a reference value of 100 , and the time is increased as the chaos parameter increases.

Since the actual travel time when the chaos parameter is 0 is 68 seconds, $112.5 \%$ when the chaos parameter is 1 means that the travel time is 76.5 seconds. Figure 8 shows snapshots over time in model (b). Figure 9 shows percentage of normalized travel time for the chaos parameter. Comparing Figure 7 with Figure 9, as the chaos parameter increases, the travel time increases qualitatively. In the case of model (a), the pedestrian has to decide which direction to go each time on the crossroad, so the travel time tends to be delayed as the chaos parameter increases. Thus, the travel time is increased by up to $112.5 \%$ from the reference 


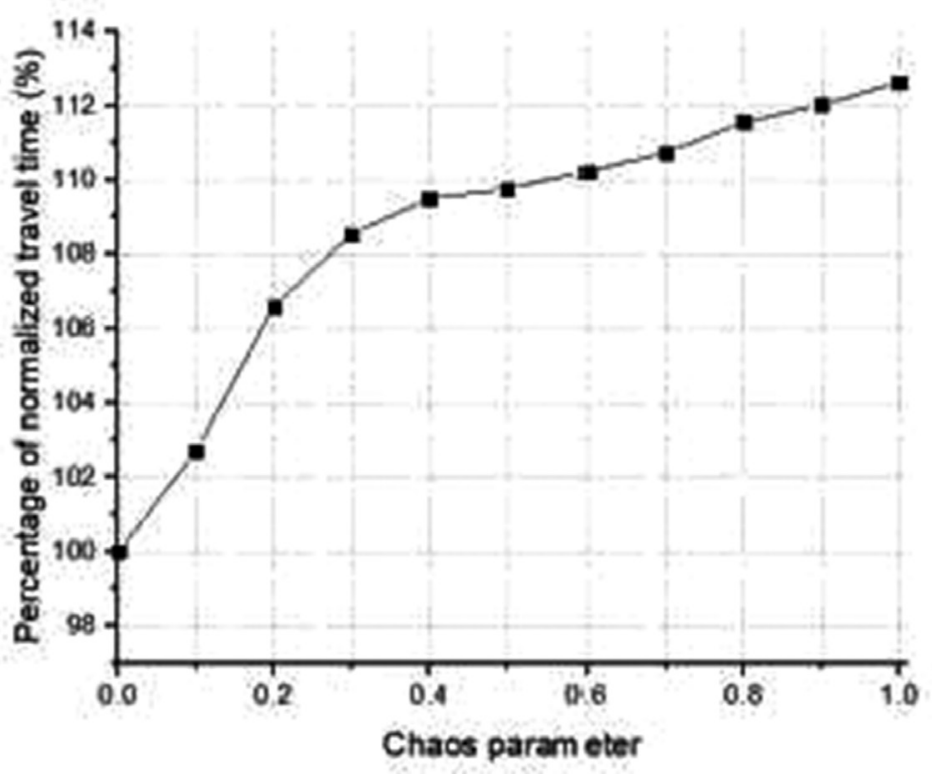

Figure 7: Travel time vs. chaos parameter for maze model a.

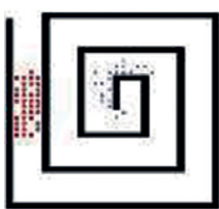

(a) $10 \mathrm{sec}$

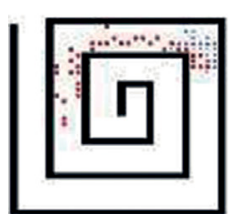

(e) $50 \mathrm{sec}$

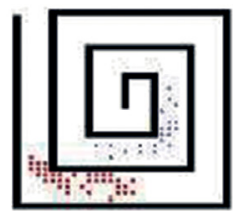

(b) $20 \mathrm{sec}$

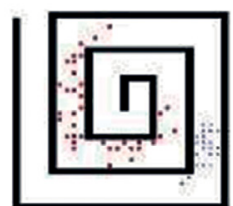

(f) $60 \mathrm{sec}$

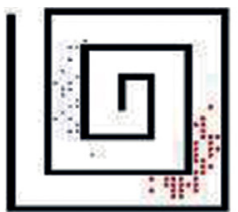

(c) $30 \mathrm{sec}$

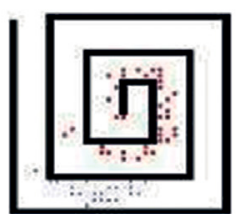

(g) $70 \mathrm{sec}$

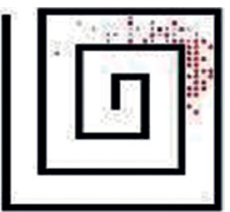

(d) $40 \mathrm{sec}$

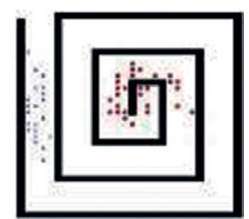

(h) $80 \mathrm{sec}$

Figure 8: Snapshots over time.

value. In model (b) without crossroads, pedestrians only find the path in the same direction, so even if the chaos parameter increases, the probability of confusion is reduced. Thus, the travel time is increased by up to $106.3 \%$ from the reference value. Since the actual travel time when the chaos parameter is 0 is 92.5 seconds, $106.3 \%$ when the chaos parameter is 1 means that the travel time is 98.3 seconds. Compared to the value of the maze model (a), it is much smaller. In other words, if the path is simple, the travel time does not increase much even if the chaos parameter increases. In the event of a disaster such as a fire, the simple structure of the multi-facility building or subway station means that it is effective in reducing people's evacuation time. 


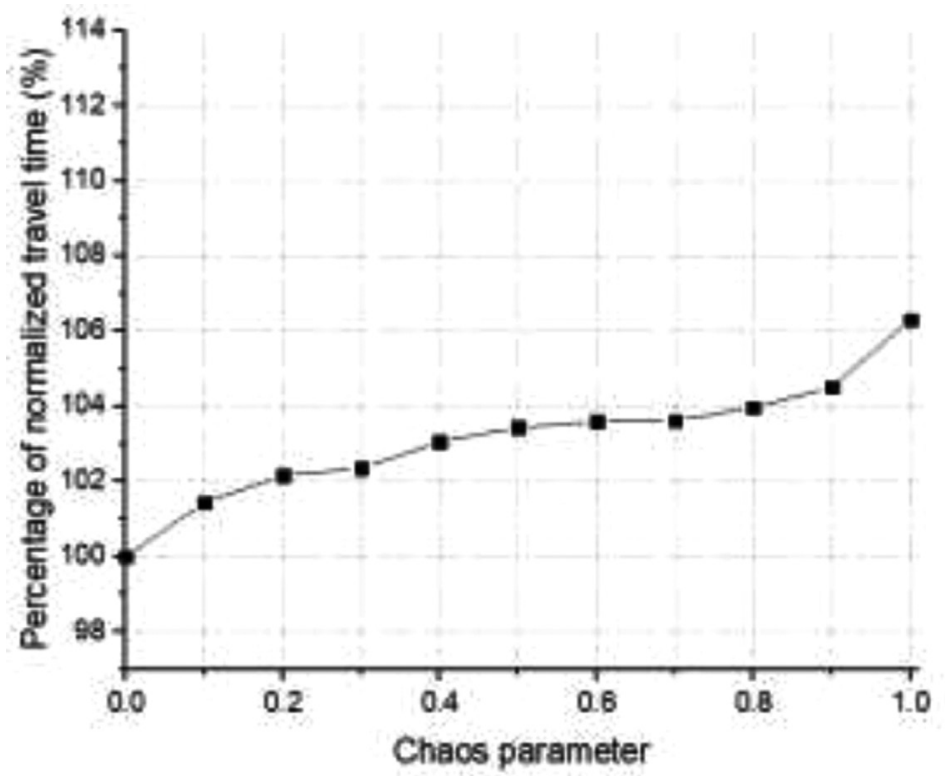

Figure 9: Travel time vs. chaos parameter for maze model b.

\section{CONCLUSIONS}

Metropolitan railway stations, terminals, multi-purpose facilities and stadiums are representative crowded places. The analysis of crowd flow is important not only for the improvement of user's path but also for the prevention of safety accidents. In this study, a new numerical analysis method was developed to simulate pedestrian flow and applied to ticket gate models of railway station and maze models. The analysis results of the ticket gate models are as follows. Depending on how the ticket gates are installed, the travel time varies significantly from case to case. It is effective to install the gates so that the paths of pedestrians are short and do not overlap between the paths. If the paths of pedestrians are not known in advance, gate model that allows both directions may be the second best. It is interesting to note that the model with gates installed according to the 'keep right' rule has the longest travel time. From these results, it is important to predict the paths of pedestrians when designing a facility or building with crowds. For the maze model, we analysed how the chaos parameter affects travel time. The analysis results of the maze models are as follows. In the model with crossroads, the pedestrian has to decide which direction to go each time on the crossroad, so the travel time tends to be delayed as the chaos parameter increases. In the model without crossroads, pedestrians only find the path in the same direction, so even if the chaos parameter increases, the probability of confusion is reduced. The maximum increase in travel time for each model is $112.5 \%$ and $106.3 \%$. It means that if the path is simple, the travel time does not increase much even if the chaos parameter increases. In the event of a disaster such as a fire, people will be in chaos, and short evacuation times, smooth flow without rushing and simple paths that do not cross each other will ensure their safety. Of course, quick rescue and firefighting are also important, but these are help from outside. Therefore, it is effective to design the multi-facility buildings or subway stations as simple as possible by optimizing so that the paths of users can be distributed without overlapping. 


\section{ACKNOWLEDGEMENTS}

This research was supported by a grant (PK2001C4) funded by National Research Council of Science \& Technology of Korean government.

\section{REFERENCES}

[1] Nam, S., Development of algorithm for passenger flow analysis based on DEM. Journal of the Korean Society for Railway, 8(4), pp.337-341, 2005.

[2] Nam, S., Crowd flow simulation using the potential path line method, WIT Transactions on Engineering Sciences, 125, pp.44-837, 2019.

[3] Helbing, D., Farkas, I. \& Vicsek, T., Simulating dynamical features of escape panic, Nature, 407(28), pp.487-490, 2000.

[4] Helbing, D., Molnar, P., Social force model for pedestrian dynamics, Physical Review E, 51, pp.4282-4286, 1995.

[5] Seyfried, A., Steffen, B. \& Lippert, T., Basics of modelling the pedestrian flow, Physica A, 368, pp.232-238, 2006.

[6] Nagal, K., Schreckenber, M., A cellular automation model for freeway traffic, Journal of Physics, 2(12), pp.2221-2229, 1992.

[7] Burstedde, C., Klauck, K. \& Schadschneider, A., Simulation of pedestrian dynamics using a two-dimensional cellular automaton, Physica A, 295, pp.507-525, 2001.

[8] Blue, V., Adler, J., Cellular automata microsimulation for modelling bi-directional pedestrian walkways, Transportation Research Part B, 35, pp.293-312, 2001.

[9] Helbing, D., Isobe, M., Nagatani, T. \& Takimoto, K., Lattice gas simulation of experimentally studied evacuation dynamics, Physical Review E, 67, 067101, pp.1-4, 2003.

[10] Guo, R., Huang, H., A mobile lattice gas model for simulating pedestrian evacuation, Physica A, 387, pp.580-586, 2008.

[11] Antonini, G., Bierlaire, M. \& Weber, M., Discrete choice models of pedestrian walking behavior, Transportation Research Part B, 40, pp.667-687, 2006.

[12] Singh, H., Arter, R., Dodd, L., Langston, P., Lester, E. \& Drury, J., Modelling subgroup behavior in crowd dynamics DEM simulation, Applied Mathematical Modelling, 33, pp.4408-4423, 2009.

[13] Heliovaara, S., Korhonen, T., Hostikka, S. \& Ehtamo, H., Counterflow model for agentbased simulation of crowd dynamics, Building and Environment, 48, pp.89-100, 2012.

[14] Lohner, R., On the modelling of pedestrian motion, Applied Mathematical Modelling, 34, pp.366-382, 2010. 